\title{
TOTAL DOMINATION VERSUS PAIRED-DOMINATION IN REGULAR GRAPHS
}

\author{
Joanna Cyman ${ }^{1}$, Magda Dettlaff ${ }^{1}$ \\ Michael A. Henning ${ }^{2 *}$, Magdalena Lemańska ${ }^{1}$ \\ AND \\ JOANNA RACZEK ${ }^{1}$ \\ ${ }^{1}$ Department of Mathematics \\ Gdansk University of Technology \\ ${ }^{2}$ Department of Pure and Applied Mathematics \\ University of Johannesburg \\ South Africa \\ e-mail: \{joana,magda,mdettlaff,gardenial\}@mif.pg.gda.pl \\ mahenning@uj.ac.za
}

\begin{abstract}
A subset $S$ of vertices of a graph $G$ is a dominating set of $G$ if every vertex not in $S$ has a neighbor in $S$, while $S$ is a total dominating set of $G$ if every vertex has a neighbor in $S$. If $S$ is a dominating set with the additional property that the subgraph induced by $S$ contains a perfect matching, then $S$ is a paired-dominating set. The domination number, denoted $\gamma(G)$, is the minimum cardinality of a dominating set of $G$, while the minimum cardinalities of a total dominating set and paired-dominating set are the total domination number, $\gamma_{t}(G)$, and the paired-domination number, $\gamma_{\text {pr }}(G)$, respectively. For $k \geq 2$, let $G$ be a connected $k$-regular graph. It is known [Schaudt, Total domination versus paired domination, Discuss. Math. Graph Theory $32(2012) 435-447]$ that $\gamma_{\mathrm{pr}}(G) / \gamma_{t}(G) \leq(2 k) /(k+1)$. In the special case when $k=2$, we observe that $\gamma_{\mathrm{pr}}(G) / \gamma_{t}(G) \leq 4 / 3$, with equality if and only if $G \cong C_{5}$. When $k=3$, we show that $\gamma_{\mathrm{pr}}(G) / \gamma_{t}(G) \leq 3 / 2$, with equality if and only if $G$ is the Petersen graph. More generally for $k \geq 2$, if $G$ has girth at least 5 and satisfies $\gamma_{\mathrm{pr}}(G) / \gamma_{t}(G)=(2 k) /(k+1)$, then we show that $G$ is a diameter-2 Moore graph. As a consequence of this result, we prove that for $k \geq 2$ and $k \neq 57$, if $G$ has girth at least 5 , then
\end{abstract}

\footnotetext{
${ }^{*}$ Research supported in part by the South African National Research Foundation and the University of Johannesburg.
} 
$\gamma_{\mathrm{pr}}(G) / \gamma_{t}(G) \leq(2 k) /(k+1)$, with equality if and only if $k=2$ and $G \cong C_{5}$ or $k=3$ and $G$ is the Petersen graph.

Keywords: domination, total domination, paired-domination.

2010 Mathematics Subject Classification: 05C69, 05 C99.

\section{REFERENCES}

[1] M. Blidia, M. Chellali and T.W. Haynes, Characterizations of trees with equal paired and double domination numbers, Discrete Math. 306 (2006) 1840-1845. doi:10.1016/j.disc.2006.03.061

[2] B. Brešar, M.A. Henning and D.F. Rall, Paired-domination of Cartesian products of graphs, Util. Math. 73 (2007) 255-265.

[3] J.A. Bondy and U.S.R. Murty, Graph Theory with Applications (North Holland, New York, 1976).

[4] X.-G. Chen, L. Sun and H.-M. Xing, Paired-domination numbers of cubic graphs, Acta Math. Sci. Ser. A Chin. Ed. 27 (2007) 166-170, in Chinese.

[5] T.C.E. Cheng, L.Y. Kang and C.T. Ng, Paired domination on interval and circulararc graphs, Discrete Appl. Math. 155 (2007) 2077-2086. doi:10.1016/j.dam.2007.05.011

[6] T.C.E. Cheng, L.Y. Kang and E. Shan, A polynomial-time algorithm for the paired-domination problem on permutation graphs, Discrete Appl. Math. 157 (2009) 262-271. doi:10.1016/j.dam.2008.02.015

[7] W.J. Desormeaux, T.W. Haynes, M.A. Henning and A. Yeo, Total domination in graphs with diameter 2, J. Graph Theory 75 (2014) 91-103. doi:10.1002/jgt.21725

[8] W.J. Desormeaux and M.A. Henning, Paired domination in graphs: a survey and recent results, Util. Math. 94 (2014) 101-166.

[9] P. Dorbec and S. Gravier, Paired-domination in $P_{5}$-free graphs, Graphs Combin. 24 (2008) 303-308. doi:10.1007/s00373-008-0792-x

[10] P. Dorbec, S. Gravier and M.A. Henning, Paired-domination in generalized claw-free graphs, J. Comb. Optim. 14 (2007) 1-7. doi:10.1007/s10878-006-9022-8

[11] P. Dorbec, B. Hartnell and M.A. Henning, Paired versus double domination in $K_{1, r^{-}}$ free graphs, J. Comb. Optim. 27 (2014) 688-694. doi:10.1007/s10878-012-9547-y

[12] O. Favaron and M.A. Henning, Paired-domination in claw-free cubic graphs, Graphs Combin. 20 (2004) 447-456.

doi:10.1007/s00373-004-0577-9 
[13] W. Goddard, personal communication at 16th CID Workshop on Graph Theory, September 20-25 (2015) Szklarska Poręba, Poland.

[14] W. Goddard and M.A. Henning,, A characterization of cubic graphs with paireddomination number three-fifths their order, Graphs Combin. 25 (2009) 675-692. doi:10.1007/s00373-010-0884-2

[15] T.W. Haynes, S.T. Hedetniemi and P.J. Slater, Fundamentals of Domination in Graphs (Marcel Dekker, New York, 1998).

[16] T.W. Haynes, S.T. Hedetniemi and P.J. Slater, Domination in Graphs: Advanced Topics (Marcel Dekker, New York, 1998).

[17] T.W. Haynes and P.J. Slater, Paired-domination and the paired-domatic number, Congr. Numer. 109 (1995) 65-72.

[18] T.W. Haynes and P.J. Slater, Paired-domination in graphs, Networks 32 (1998) 199-206.

doi:10.1002/(SICI)1097-0037(199810)32:3〈199::AID-NET4〉3.0.CO;2-F

[19] M.A. Henning, Graphs with large paired-domination number, J. Comb. Optim. 13 (2007) 61-78. doi:10.1007/s10878-006-9014-8

[20] M.A. Henning, A survey of selected recent results on total domination in graphs, Discrete Math. 309 (2009) 32-63.

doi:10.1016/j.disc.2007.12.044

[21] M.A. Henning and A. Yeo, Total Domination in Graphs (Springer Monographs in Mathematics, Springer-Verlag, New York, 2013).

[22] A.J. Hoffman and R.R. Singleton, On Moore graphs with diameter 2 and 3, IBM J. Res. Dev. 4 (1960) 497-504. doi: $10.1147 / \mathrm{rd} .45 .0497$

[23] O. Schaudt, Total domination versus paired domination, Discuss. Math. Graph Theory 32 (2012) 435-447. doi:10.7151/dmgt.1614

[24] N. Robertson, Graphs Minimal Under Girth, Valency, and Connectivity Constraints (Dissertation, Waterloo, Ontario, University of Waterloo, 1969).

[25] R.R. Singleton, There is no irregular Moore graph, Amer. Math. Monthly 75 (1968) 42-43. doi: $10.2307 / 2315106$

Received 31 August 2016

Revised 11 January 2017

Accepted 11 January 2017 